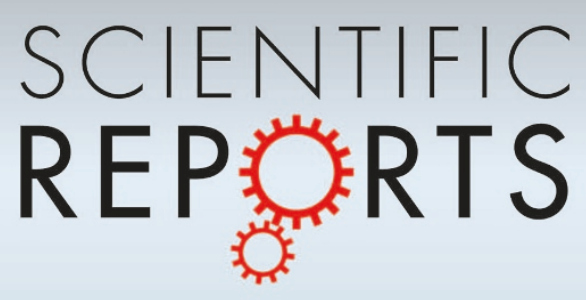

\title{
OPEN A General Strategy for Hybrid Thin Film Fabrication and Transfer onto Arbitrary
}

SUBJECT AREAS:

ELECTRONIC PROPERTIES

AND DEVICES

SYNTHESIS AND PROCESSING

TWO-DIMENSIONAL MATERIALS

OPTICAL PROPERTIES AND

DEVICES

\section{Substrates}

\author{
Yong Zhang, John J. Magan \& Werner J. Blau
}

School of Physics and Centre for Research on Adaptive Nanostructures and Nanodevices (CRANN), Trinity College Dublin, Dublin 2 , Ireland.

Received

21 February 2014

Accepted

10 April 2014

Published

28 April 2014

Correspondence and requests for materials should be addressed to Y.Z. (zhangyo@tcd.ie) or W.B. (wblau@tcd.

The development of thin film-based structures/devices often requires thin films to be transferred onto arbitrary substrates/surfaces. Controllable and non-destructive transfer method, although highly desired, remains quite challenging. Here we report a general method for fabrication and transfer of hybrid (ultra)thin films. The proposed solution-based in-situ transfer method shows not only its robust ability for thin film transfer onto arbitrary substrates but also its highly controlled and non-destructive characteristic. With a hole structure as the support, fully-stretched free-standing thin film is prepared. The successful transfer to a curved surface demonstrates the possibility for production of thin film-coated complex optical components. Ultrathin $(35 \mathrm{~nm})$ hybrid film transferred onto PET (50 $\mu \mathrm{m}$ thick) shows high transparency $\left(>90 \%\right.$ in visible range), conductivity $\left(1.54 \times 10^{4} \mathrm{~S} / \mathrm{m}\right)$, and flexibility (radius of curvature down to $\mathrm{mm}$ scale). The reported transfer method would provide a powerful route towards complex thin film-based structures/devices.

T hin film-based nanomaterials are attracting much attention owning to its solid state, low-dimension, and large specific surface area. Hybrid thin/ultrathin (i.e., with thickness less than $100 \mathrm{~nm}$ ) films incorporating various functional components can be utilized into cutting-edge research and applications such as magnetism, optics, electronics, and catalysis ${ }^{1-9}$. Current industrial trends go towards flexible and printable electronics and photonics ${ }^{5,6}$. It has been shown that carbon nanotube (CNT)-based thin films are promising candidates for such applications $s^{7,8}$. Vacuum filtration, among many production methods ${ }^{7,8,10-16}$, has been widely used for fabrication of thin films due to its high and reproducible film quality and production efficiency ${ }^{7,8,10}$. However, such thin films have often to be transferred onto desired substrates prior to its practical applications ${ }^{17}$. So far, thin film transfer still remains quite challenging especially onto arbitrary substrates/surfaces ${ }^{18}$. Polydimethylsiloxane (PDMS) stamp transfer method ${ }^{10,13,19,20}$, although is a useful technique, suffers from a few drawbacks, e.g., the difficulty to transfer onto arbitrary substrates such as hole structures or curved surfaces and the unavoidable direct contact between the stamp and the film. Another notable transfer method, which is based on solventetchable organic (e.g., mixed cellulose ester (MCE)) filter membranes, has received much success on fabrication and transfer of carbon-based thin films ${ }^{5,6,8,21}$. However the major drawback is that this method can only be applied onto aqueous dispersion systems, e.g., CNTs or graphene dispersed in water $\left(\mathrm{H}_{2} \mathrm{O}\right)$ with surfactant. Heat and pressure process is often applied to improve the quality of the transferred film, indicating that this approach is relatively destructive. It is also challenging to completely remove the surfactants from the thin film which restricts its applications in high-performance structures/devices. On the other hand, with the recent great success of solvent-exfoliation of graphite and other layered materials for mass-production of high-quality graphene and other 2D monolayers ${ }^{22-24}$, the increasingly urgent need for developing new fabrication and transfer method towards thin films from non-aqueous dispersions, e.g., CNTs in n-cyclohexyl-2-pyrrolidone (CHP) or graphene in n-methyl-2-pyrrolidone (NMP), greatly prompts the emergence of this work.

Here we report a general strategy for fabrication and transfer of hybrid (ultra)thin films towards non-aqueous dispersion systems. We introduce a solution-based in-situ transfer method which showed its highly controlled and non-destructive characteristic when applied to arbitrary substrates/surfaces such as hole structures, curved surfaces, glass, polyethylene terephthalate (PET), and flexible substrates (e.g., $50 \mu \mathrm{m}$ thick PET films). As a demonstration of the reported method, CNT-based and graphene-based hybrid thin films were fabricated, transferred, and characterized. More complex thin film incorporating graphene and dye molecule (i.e., 5, 10, 15,20 -tetraphenyl- $21 \mathrm{H}, 23 \mathrm{H}$-porphine) aggregates was also fabricated and transferred and could be interesting as 


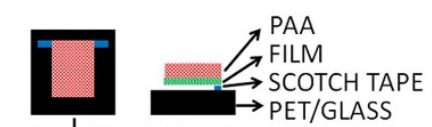

TOP- $Y I E W$ SIDE-VIEW

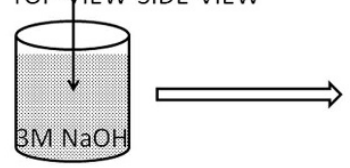

1) PAA ETCHING

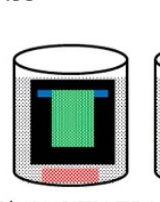

2) IN-SITU TRANSFER
TRANSFER INTO WATER

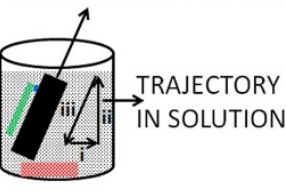

西

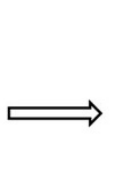

3) WASHING
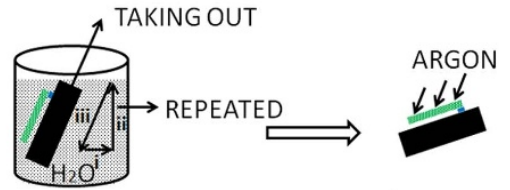

4) DRYING

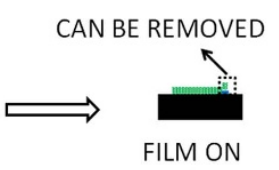

PET/GLASS

Figure 1 | Schematic illustration towards the general method for in-situ transfer of hybrid (ultra)thin films.

novel nonlinear optical (NLO) materials. The CNT-based ultrathin $(35 \mathrm{~nm}$ ) hybrid film shows broad-band high transparency ( $>90 \%$ in visible and $>80 \%$ in near infra-red (NIR) range) and conductivity $\left(1.54 \times 10^{4} \mathrm{~S} / \mathrm{m}\right)$ which indicate its potential applications as transparent conductive electrodes. The transferred ultrathin films are durable and highly solvent-resistant which promise its practical applications.

\section{Results}

Figure S2 demonstrates the fabrication process of hybrid (ultra)thin films supported on porous anodic alumina (PAA) membranes. It should be noted that all of the thin films presented in this work are hybrid films using poly(3,4-ethylenedioxythiophene) doped with poly(styrenesulfonate) (PEDOT:PSS) as matrices. PEDOT:PSS is a conjugate polymer that is widely used in transparent conductive electrodes. Experimental details can be found in the Supplementary Information. Other polymers such as polystyrene (PS), poly (methyl methacrylate) (PMMA), and polyvinyl alcohol (PVA) can be used as matrices depending on the applications. Polymer matrix not only promises flexible processability but also provides mechanical support to the hybrid thin film that is required in the solution-based transfer and washing processes. Unless stated otherwise, commercially available $20 \mathrm{~nm}$ (pore size) PAA membranes were used for vacuum filtration. Home-made PAA membranes (with pore size down to $5 \mathrm{~nm}$ ) could be used under certain circumstances. Such PAA-based vacuum filtration is, in principle, suitable for any component with at least one dimension larger than $20 \mathrm{~nm}$ (pore size) $)^{10,19}$. This method is also benefited from the possible large-area and highthroughput production of thin films with controlled thickness. Insitu and post washing the thin films with volatile solvents such as ethanol would efficiently remove the remaining high-boiling-point dispersants, e.g., CHP or NMP, which facilitates the production of high-performance thin film-based structures/devices. A general method for in-situ transfer of the as-fabricated hybrid thin films was illustrated in Figure 1. Sodium hydroxide $(\mathrm{NaOH})$ aqueous solution was applied to etch away the PAA membranes. Prior to the etching step, a specific configuration of the samples was adopted. As shown in Figure 1, the film on PAA was placed upside down onto the desired substrates, e.g., PET or glass, with one edge attached onto the support through double-sided scotch tape. Such simple but unique configuration enables the flexible fixation of the free-standing thin films on the substrates after removal of the PAA membranes. Another key function of such line-contact mode is to ensure that the free-standing thin films were directionally guided throughout the whole solution process, e.g., etching and washing step. It should be noted that $3 \mathrm{M} \mathrm{NaOH}$ solution was used in order to quickly (in 10 seconds) detach the PAA membranes from the thin films, making the etching process very efficient. Furthermore, to keep the solution process safe and sufficient, a specific and repeatable trajectory, as shown in Figure 1, was introduced. A tilting posture, rather than vertical placement, was adopted for both the putting-in and taking-out step during etching and washing. To ensure that no residual $\mathrm{NaOH}$ was left on/in the thin films, greatly excess amount of deionized water was used for the washing step. In specific, the hybrid thin films were washed sequentially in three filled large beakers $(500 \mathrm{~mL})$ and with three times for each beaker at a relatively slow movement along the trajectory (e.g., 3-5 seconds per cycle). The following drying process with argon is also delicate and cautions must be taken to avoid bubbles and wrinkles. Practice would be very helpful to make bubble-free and wrinkle-free transferred thin films ${ }^{25}$. Such bubble and wrinkle issues could also occur during the above solution process. For this reason, a volume ratio $(1: 4)$ of ethanol to the aqueous solution was used to minimize the bubble and wrinkle issues, in case of PET substrates. Now the hybrid thin films have been transferred onto the desired substrates. When necessary, the scotch tape can be removed from PET or glass using cotton with ethanol or razor blade respectively.

Based on the proposed method, hybrid thin films on arbitrary substrates/surfaces were obtained. Figure 2 shows the photographs of various transferred thin films. Thin film transferred onto a hole structure, as illustrated in Figure 2a, can function as free-standing thin films (in the unsupported hole area) which could be very useful in substrate-free applications. It should be noted that flow drying is not suitable in case of a hole structure. An alternative method, i.e., natural drying when taken out from pure ethanol, was used. Fully self-stretched free-standing thin film was formed after the ethanol was rapidly and completely evaporated under ambient conditions. The ultrathin film transferred onto PET (50 $\mu \mathrm{m}$ thick) (Figure 2b) shows promising properties such as high transparency (Figure 2c) and flexibility (radius of curvature down to $\mathrm{mm}$ scale, Figure $2 \mathrm{~d}$ ), which will be detailed in the following sections. Thin film transferred onto curved surface is shown in Figure 2e. This suggests the possibility for production of thin film-coated complex optical components (e.g., hole/dot/rod arrays), which could be very useful in lasering,

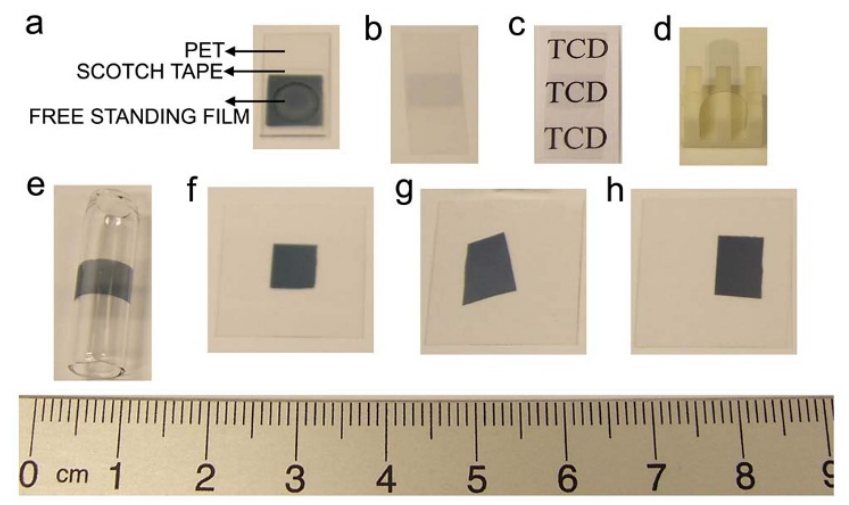

Figure $2 \mid$ Photographs for demonstration of the universality of the proposed method. a-f) CNT-based hybrid thin films. a) Free-standing thin film covering a $5 \mathrm{~mm}$ hole in PET. b) Ultrathin film transferred onto PET (50 $\mu \mathrm{m}$ thick) showing its high transparency (c) and flexibility (d).e) Thin film transferred onto curved surface of glass. f) Thin film transferred onto glass. g) Graphene-based hybrid thin film transferred onto glass. h) Complex thin film incorporating graphene and dye molecule aggregates transferred onto glass. 


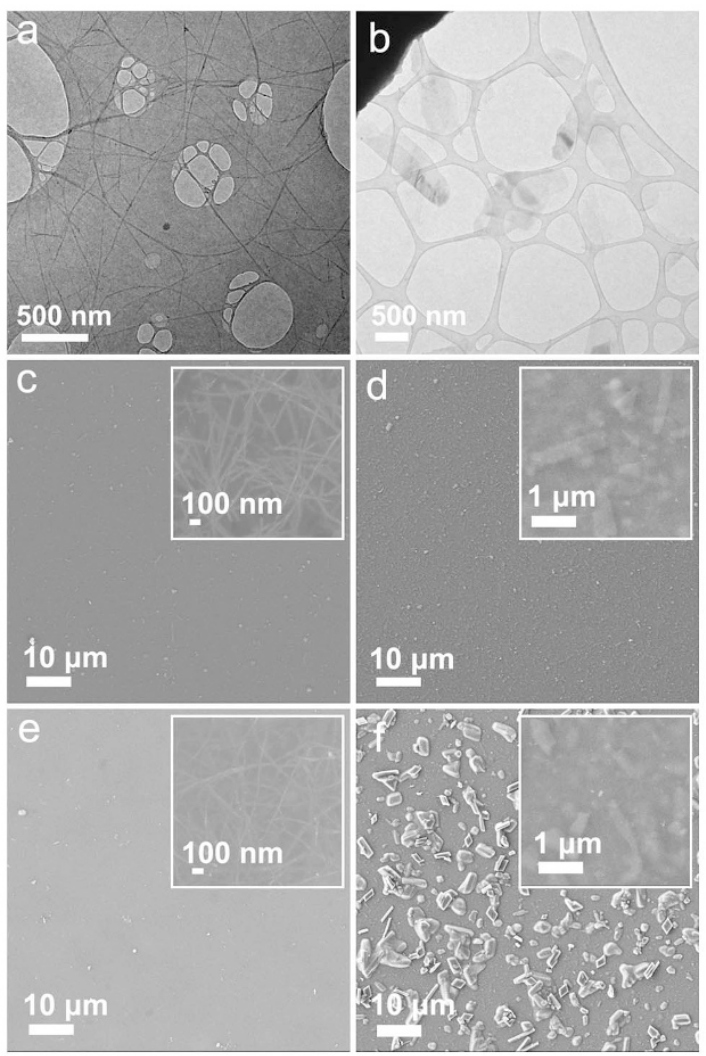

Figure 3 Microscopic characterization of the CNTs, graphene, and their corresponding hybrid (ultra)thin films. a) TEM image of CNTs from its dispersion system. b) TEM image of graphene from its dispersion system. $c$ and e) FE-SEM images of the CNT-based thin and ultrathin films shown in Figure $2 b$ and $2 f$ respectively. d) FE-SEM image of the graphene-based thin film shown in Figure 2g. f) FE-SEM image of the complex thin film incorporating graphene and dye molecule aggregates shown in Figure $2 \mathrm{~h}$. The insets show high-magnification images.

imaging, and sensing ${ }^{26}$. Thin films can be transferred onto glass, as indicated in Figure 2f. Not only CNT-based (Figure 2a-f) but also graphene-based (Figure $2 \mathrm{~g}$ ) thin films were successfully fabricated and transferred. The proposed method applies to other 1D (e.g., molecular, polymeric, metallic, and metal oxide wires) and 2D (e.g., molybdenum disulphide $\left(\mathrm{MoS}_{2}\right)$, bismuth selenide $\left(\mathrm{Bi}_{2} \mathrm{Se}_{3}\right)$, and boron nitride $(\mathrm{BN})$ monolayers) materials, as well as $0 \mathrm{D}$ (larger than $20 \mathrm{~nm}$ ) materials, therefore could provide a powerful route for production of more complex thin films. For example, complex thin film incorporating graphene and dye molecule aggregates is presented in Figure $2 \mathrm{~h}$. Its complexity (or structural hierarchy) is clearly shown in Figure $3 f$.

The dispersion systems play an important role on the qualitycontrol of the resulting hybrid thin films. Transmission electron microscope (TEM) was used to characterize the CNT and graphene dispersions which were made as described elsewhere ${ }^{27,28}$. Figure 3a and $3 \mathrm{~b}$ clearly reveal that both CNTs and graphene are well-dispersed on the supporting copper grids. It is known that aggregation often occurs after the solvents are completely evaporated. In our case, no bundles of CNTs or bulk graphite were found by searching the entire copper grids. They are mostly individual CNTs or monolayer and few-layer graphene, indicating the high quality of the dispersion systems. The thin films formed from these dispersions were characterized by field emission scanning electron microscope (FE-SEM). It should be noted that all SEM measurements were done without metallic coatings owning to the conductivity of the hybrid thin films. To avoid the charging issue during SEM measurements, both ends of the thin films were connected to the conducting stage through double-sided conductive carbon tape. Therefore these SEM images present the real surfaces of the thin films. Figure S3 presents the effect of metallic (i.e., $\mathrm{Au}$ ) coating on the SEM images of the complex thin film incorporating graphene and dye molecule aggregates (the same sample as in Figure 2h). Figure $3 \mathrm{c}$ and $3 \mathrm{e}$ reveal that the surfaces of the CNT-based thin and ultrathin films are quite uniform over large areas, consistent with that observed from the photographs (Figure $2 \mathrm{~b}$ and $2 \mathrm{f}$ ) of the thin films. To reveal the CNT networks on the surfaces of the thin films, InLens detector was used, as shown in the insets of Figure $3 \mathrm{c}$ and $3 \mathrm{e}$. Figure S4 presents the direct comparison of the images taken from InLens and SE (secondary electron) detector respectively. The surface of graphene-based thin film, as presented in Figure 3d, is also very uniform over wide ranges. The inset indicates that graphene sheets are well-distributed on the surface of the thin film. Compared with graphene-based thin film (Figure 3d), more complex thin film (Figure 3f) shows relatively rough surface with well-distributed dye molecule aggregates. Similar situation on graphene distribution (inset of Figure 3f) was found in this complex thin film.

As mentioned above, one of the promising applications for the hybrid thin films is transparent conductive electrodes. Figure 4a presents the absorption spectra of the thin films as shown in Figure $2 b$, $2 \mathrm{f}$, and $2 \mathrm{~g}$. The significantly low absorbance of the ultrathin film (Figure 2b) indicates its high transparency. As shown in Figure $4 \mathrm{~b}$, the transmittance of the ultrathin film is above $90 \%$ in the visible range and above $80 \%$ in the NIR range. In particular, the transmittance at $550 \mathrm{~nm}$ is above $94 \%$, suggesting its extraordinarily high transparency. Figure $4 \mathrm{c}$ presents the I-V curve from the 4-probe electrical measurement of the ultrathin film. The linear behaviour of the measured I-V response indicates that such ultrathin film is suitable as electrode materials. The sheet resistance was measured to be $1850 \Omega / \square$, higher than the industry standard (i.e., $100-200 \Omega / \square$ ). However, the conductivity was calculated to be as high as $1.54 \times$ $10^{4} \mathrm{~S} / \mathrm{m}$ based on the ultrathin ( $35 \mathrm{~nm}$, see Figure S5) feature of the film. Therefore, the ultrathin film could be used as transparent conductive electrodes for applications such as solar cell. One of the key parameters for practical applications is the durability of the thin films. Figure $4 \mathrm{~d}$ demonstrates that the ultrathin film is highly solvent-resistant. A simple test (i.e., applying the scotch tape on the film, pressing hard, and slowly removing the tape from the substrate) reveals the relatively strong adhesion between the ultrathin film and the supporting PET film (50 $\mu \mathrm{m}$ thick). It should be noted that no observable damage on the ultrathin film was found after several cycles of rubbing the surface with gloved fingers (with nitrile gloves), indicating such ultrathin film is also durable.

It should be noted that a dry transfer technique was recently reported $^{29}$, which offers an extremely simple (direct contact and press transfer) and rapid $(<15 \mathrm{~s})$ transfer method towards largescale $\left(28 \times 28 \mathrm{~cm}^{2}\right)$ CNT networks. However such a dry transfer technique could suffer from similar drawbacks to the PDMS method, e.g., the difficulty to transfer onto arbitrary substrates such as hole structures or curved surfaces. In addition, the transferred film could show relatively weak durability, which might be improved by a post heat and pressure process. Furthermore, the success of such a direct contact transfer process was determined by not only the relative adhesive strength of the film on the target and source substrates but also the mechanical strength of the film, indicating the limited applications in such as 1D nanostructure/nanomaterial networks. Our solution-based in-situ transfer method overcomes the above drawbacks and provides a powerful technique towards diverse substrates, durable transferred films, and broad available film components. Both solution-based and dry transfer methods can be utilized for applications such as transparent, conductive, and flexible electrodes. For example, the ultrathin CNT hybrid film showed a relatively low sheet resistance of $1850 \Omega / \square$ at a very high transmittance of 

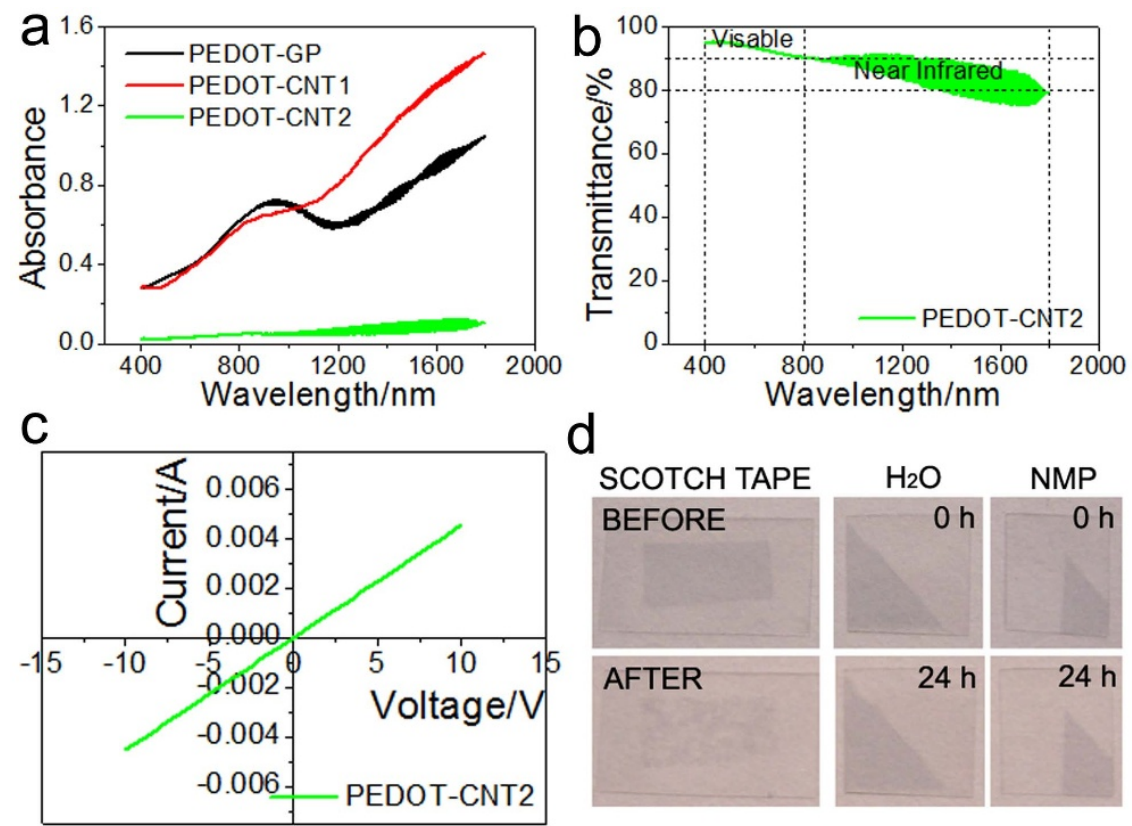

d

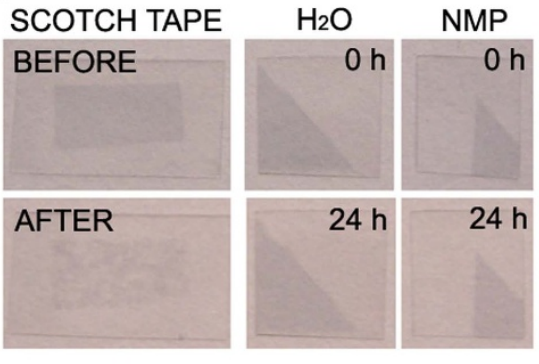

Figure $4 \mid$ Optical and electrical characterization and durability test of the hybrid (ultra)thin films. a) Absorption spectra of thin films shown in Figure 2b, 2f, and 2g respectively. b) Transmission spectrum of the ultrathin film shown in Figure 2b. c) I-V curve of the ultrathin film shown in Figure 2b. d) Durability test of the ultrathin film shown in Figure 2b.

$94 \%$, similar to that $(2700 \Omega / \square$ at $90 \%)$ of the intrinsic CNT $(3.3 \mu \mathrm{m})$ networks. The sheet resistance of the treated CNT networks was significantly decreased to as low as $110 \Omega / \square$, approaching the industry standard.

\section{Conclusion}

In summary, we have described a general method towards fabrication and transfer of hybrid (ultra)thin films onto arbitrary substrates/ surfaces. The solution-based in-situ transfer route is safe, efficient, and fast, which indicate its potential for large-scale production of high-quality thin films on desired substrates. Ultrathin $(35 \mathrm{~nm})$ film transferred onto PET ( $50 \mu \mathrm{m}$ thick) demonstrates high transparency $(94 \%$ at $550 \mathrm{~nm})$, conductivity $\left(1.54 \times 10^{4} \mathrm{~S} / \mathrm{m}\right)$, and flexibility (radius of curvature down to $\mathrm{mm}$ scale) which could be promising in applications such as flexible electronics, touch screen, and solar cells. The demonstration of thin films transferred onto hole structures and curved surfaces suggests its bright future in imaging and sensing applications. The reported method could also be used for development of novel and complex thin film-based NLO components for advanced laser-based technologies. It is expected that layer-by-layer technique could be simply employed in the proposed method to produce multi-layered thin film-based structures/devices.

\section{Methods}

Fabrication and transfer of the hybrid thin films. Vacuum filtration was applied to fabricate the hybrid thin films using poly(3,4-ethylenedioxythiophene) doped with poly(styrenesulfonate) (PEDOT:PSS) as the matrices. PEDOT:PSS was purchased from Heraeus under the trade name Clevios PH 500, with initial concentration of 10$13 \mathrm{mg} / \mathrm{mL}$ in water. Dye molecules (i.e., 5, 10, 15, 20-tetraphenyl-21H, 23H-porphine $(\mathrm{PPH})$ ) were purchased from Sigma Aldrich and used as received. To fabricate the complex thin film incorporating graphene and dye molecule aggregates, $\mathrm{PPH}$ was dissolved in NMP at a concentration of $0.05 \mathrm{mg} / \mathrm{mL}$. The stock CNT and graphene dispersions were with concentration of $0.36 \mathrm{mg} / \mathrm{mL}$ and $0.4 \mathrm{mg} / \mathrm{mL}$ respectively. For CNT-based thin films, PEDOT:PSS/ $\mathrm{H}_{2} \mathrm{O}(50 \times$ (i.e., 50 times diluted), $1 \mathrm{~mL})$ and SWCNT/CHP $(45 \times, 1 \mathrm{~mL})$ were mixed for vacuum filtration. For CNT-based ultrathin films, PEDOT:PSS/ $\mathrm{H}_{2} \mathrm{O}(500 \times, 1 \mathrm{~mL})$ and SWCNT/CHP $(450 \times, 1 \mathrm{~mL})$ were mixed for vacuum filtration. For graphene-based thin films, PEDOT:PSS/ $\mathrm{H}_{2} \mathrm{O}$ $(50 \times, 1 \mathrm{~mL})$ and graphene/NMP $(50 \times, 1 \mathrm{~mL})$ were mixed for vacuum filtration. Note that the above mixtures were homogeneous systems within a period of up to $24 \mathrm{~h}$, sufficient for vacuum filtration which usually took up to $30 \mathrm{~min}$. For complex thin films, PEDOT:PSS/ $\mathrm{H}_{2} \mathrm{O}(50 \times, 1 \mathrm{~mL})$, graphene/NMP $(50 \times, 1 \mathrm{~mL})$, and $\mathrm{PPH} /$ NMP $(0.05 \mathrm{mg} / \mathrm{mL}, 1 \mathrm{~mL})$ were mixed for vacuum filtration. It should be noted that a turbid system was obtained when mixing the three components, due to the formation of the dye molecule aggregates. Solution-based in-situ transfer method was applied for transfer of the hybrid thin films onto arbitrary substrates. Details can be found in the Results Section.

Characterization. The photographs of the hybrid thin films were taken by a digital camera. The hybrid thin films were characterized by field-emission scanning electron microscopy (FE-SEM, Zeiss Ultra Plus) at an acceleration voltage of $5 \mathrm{kV}$, transmission electron microscopy (TEM, JEOL 2100) at an acceleration voltage of $200 \mathrm{kV}$, and film-based absorption spectroscopy (Agilent Cary 6000i) through a $5 \mathrm{~mm}$ diameter hole (note that all samples were larger than the hole). Sheet resistance measurements were made using the 4-probe technique with a Keithley 2400 source meter.

1. Gregg, J. F. et al. Giant magnetoresistive effects in a single element magnetic thin film. Phys. Rev. Lett. 77, 1580-1583 (1996).

2. Xi, J. Q. et al. Optical thin-film materials with low refractive index for broadband elimination of Fresnel reflection. Nature Photon. 1, 176-179 (2007).

3. Henari, F. Z., Weldon, D. N. \& Blau, W. J. Nearly resonant third-order optical non-linearity in $\mathrm{c}_{60}$ cast films. Adv. Mater. Opt. Electron. 4, 413-416 (1994).

4. Doyle, J. J. et al. Nonlinear optical performance of chemically tailored phthalocyanine-polymer films as solid-state optical limiting devices. J. Opt. A: Pure Appl. Opt. 10, 075101 (2008).

5. De, S. et al. Transparent, flexible, and highly conductive thin films based on polymer-nanotube composites. ACS Nano 3, 714-720 (2009).

6. De, S. et al. Silver nanowire networks as flexible, transparent, conducting films: extremely high DC to optical conductivity ratios. ACS Nano 3, 1767-1774 (2009).

7. Kivistoe, S. et al. Carbon nanotube films for ultrafast broadband technology. Opt. Express 17, 2358-2363 (2009).

8. Wu, Z. et al. Transparent, conductive carbon nanotube films. Science 305, 1273-1276 (2004).

9. Gong, K., Du, F., Xia, Z., Durstock, M. \& Dai, L. Nitrogen-doped carbon nanotube arrays with high electrocatalytic activity for oxygen reduction. Science 323, 760-764 (2009).

10. Zhang, D. et al. Transparent, conductive, and flexible carbon nanotube films and their application in organic light-emitting diodes. Nano Lett. 6, 1880-1886 (2006).

11. Liu, Q., Fujigaya, T., Cheng, H. M. \& Nakashima, N. Free-standing highly conductive transparent ultrathin single-walled carbon nanotube films. J. Am. Chem. Soc. 132, 16581-16586 (2010).

12. Hellstrom, S. L., Lee, H. W. \& Bao, Z. Polymer-assisted direct deposition of uniform carbon nanotube bundle networks for high performance transparent electrodes. ACS Nano 3, 1423-1430 (2009).

13. Meitl, M. A. et al. Solution casting and transfer printing single-walled carbon nanotube films. Nano Lett. 4, 1643-1647 (2004).

14. Saran, N. et al. Fabrication and characterization of thin films of single-walled carbon nanotube bundles on flexible plastic substrates. J. Am. Chem. Soc. 126, 4462-4463 (2004). 
15. Abe, Y., Tomuro, R. \& Sano, M. Highly efficient direct current electrodeposition of single-walled carbon nanotubes in anhydrous solvents. Adv. Mater. 17, 2192-2194 (2005).

16. Li, X. et al. Large-area synthesis of high-quality and uniform graphene films on copper foils. Science 324, 1312-1314 (2009).

17. Hennrich, F. et al. Preparation, characterization and applications of free-standing single walled carbon nanotube thin films. Phys. Chem. Chem. Phys. 4, 2273-2277 (2002).

18. Suk, J. W. et al. Transfer of CVD-grown monolayer graphene onto arbitrary substrates. ACS Nano 5, 6916-6924 (2011).

19. Zhou, Y., Hu, L. \& Gruener, G. A method of printing carbon nanotube thin films. Appl. Phys. Lett. 88, 123109 (2006).

20. Song, J. et al. A general method for transferring graphene onto soft surfaces. Nature Nanotech. 8, 356-362 (2013).

21. Eda, G., Fanchina, G. \& Chhowalla, M. Large-area ultrathin films of reduced graphene oxide as a transparent and flexible electronic material. Nature Nanotech. 3, 270-274 (2008).

22. Hernandez, Y. et al. High-yield production of graphene by liquid-phase exfoliation of graphite. Nature Nanotech. 3, 563-568 (2008).

23. Coleman, J. N. et al. Two-dimensional nanosheets produced by liquid exfoliation of layered materials. Science 331, 568-571 (2011).

24. Nicolosi, V., Chhowalla, M., Kanatzidis, M. G., Strano, M. S. \& Coleman, J. N. Liquid exfoliation of layered materials. Science 340, 1226419 (2013).

25. Kim, F., Cote, L. J. \& Huang, J. Graphene oxide: surface activity and twodimensional assembly. Adv. Mater. 22, 1954-1958 (2010)

26. Barnes, W. L., Dereux, A. \& Ebbesen, T. W. Surface plasmon subwavelength optics. Nature 424, 824-830 (2003).

27. Bergin, S. D., Sun, Z., Streich, P., Hamilton, J. \& Coleman, J. N. New solvents for nanotubes: approaching the dispersibility of surfactants. J. Phys. Chem. C 114, 231-237 (2010).

28. Khan, U., O’Neill, A., Lotya, M., De, S. \& Coleman, J. N. High-concentration solvent exfoliation of graphene. Small 6, 864-871 (2010).
29. Kaskela, A. et al. Aerosol-synthesized SWCNT networks with tunable conductivity and transparency by a dry transfer technique. Nano Lett. 10, 4349-4355 (2010).

\section{Acknowledgments}

This work was supported by Science Foundation Ireland through SFI Investigator Award with grant no. 12/IA/1306.

\section{Author contributions}

Y.Z. and W.B. conceived the idea and designed the experiments. Y.Z. performed the experiments. J.J.M. contributed experimental tools including the vacuum filtration and 4-probe setup. Y.Z. and W.B. analyzed the data and wrote the manuscript. All authors discussed and approved the results in the manuscript.

\section{Additional information}

Supplementary information accompanies this paper at http://www.nature.com/ scientificreports

Competing financial interests: The authors declare no competing financial interests.

How to cite this article: Zhang, Y., Magan, J.J. \& Blau, W.J. A General Strategy for Hybrid Thin Film Fabrication and Transfer onto Arbitrary Substrates. Sci. Rep. 4, 4822; DOI:10.1038/srep04822 (2014)

This work is licensed under a Creative Commons Attribution-NonCommercialNoDerivs 3.0 Unported License. The images in this article are included in the article's Creative Commons license, unless indicated otherwise in the image credit; if the image is not included under the Creative Commons license, users will need to obtain permission from the license holder in order to reproduce the image. To view a copy of this license, visit http://creativecommons.org/licenses/by-nc-nd/3.0/ 